\title{
Reproducing Kernel Methods for Solving Inverse Problems
}

\section{Bo Han* and Wenyan Wang}

Department of Mathematics, Harbin Institute of Technology, Harbin 150001, PR China

Inverse problems arise in scientific and technical fields such as geophysics, life science, remote sensing technology, pattern recognition, financial science, etc. Therefore, inverse problems have attracted much attention and have been studied by many authors $[1,2]$. However it is usually difficult to solve inverse problems. In particular, most inverse problems are very complex with a large amount of calculation. Thus, it is very important to obtain the algorithm to solve inverse problems with advantages such as high precision, little calculation, good convergence and strong stability. Good properties of reproducing kernel in calculation made it an important function approximation technique. In recent years, reproducing kernel theory is developing very rapidly, and successfully applied to wavelet transforms, signal processing, stochastic processes, iris recognition, neural network, and so on.

Recently we have successfully applied the reproducing kernel theory to inverse heat problems, two dimensional parabolic inverse problems with final over determination, and inverse source problems of two-dimensional fractional diffusion equations. And we improved the precision, decreased considerably the runtime. Numerical simulation experiments and error analyses indicate that the reproducing kernel method has many advantages as follows: numerical programming structure is simple, calculations are very fast, precision is very high, convergence is very good, and stability is strong.

However, it is well known inverse problems are always ill-posed. For the stable numerical reconstructions of solutions, regularization techniques can be used. Castro et al. [3], T. Takeuchi and Yamamoto [4] have obtained some better results combining regularization with reproducing kernels. Further, we will combine the advantages of regularization and reproducing kernels to solve more general inverse problems, and we will discuss in a forthcoming paper.

\section{References}

1. Dou YX, Han B (2012) Total variation regularization for the reconstruction of a mountain topography. Appl Numer Math 62: 1-20.

2. Xu X, Cheng J, Yamamoto M (2011) Carleman estimate for a fractional diffusion equation with half order and application. Appl Anal 90: 1355-1371.

3. Castro LP, Chen Q, Saitoh S (2010) Source inversion of heat conduction from a finite number of observation data. Appl Anal 89: 801-813.

4. Takeuchi T, Yamamoto M (2008) Tikhonov regularization by a reproducing kernel Hilbert space for the Cauchy problem for an elliptic equation. SIAM J Sci Comput 31: 112-142
*Corresponding author: Bo Han, Department of Mathematics, Harbin Institute of Technology, Harbin 150001, PR China, E-mail: bohan@hit.edu.cn

Received June 28, 2012; Accepted June 30, 2012; Published July 04, 2012

Citation: Han B, Wang W (2012) Reproducing Kernel Methods for Solving Inverse Problems. J Applied Computat Mathemat 1:e112. doi: 10.4172/2168-9679.1000e112

Copyright: $(2012$ Han B, et al. This is an open-access article distributed under the terms of the Creative Commons Attribution License, which permits unrestricted use, distribution, and reproduction in any medium, provided the original author and source are credited. 